\title{
New experimental limits on neutron - mirror neutron oscillations in the presence of mirror magnetic field
}

\author{
Z. Berezhiani ${ }^{1,2, a}$, R. Biondi ${ }^{1,2}$, P. Geltenbort ${ }^{3}$, I. A. Krasnoshchekova ${ }^{4}$, V. E. Varlamov $^{4}$, A. V. Vassiljev ${ }^{4}$, \\ O. M. Zherebtsov ${ }^{4}$ \\ ${ }^{1}$ Dipartimento di Fisica e Chimica, Università di L'Aquila, 67100 Coppito, L'Aquila, Italy \\ 2 INFN, Laboratori Nazionali del Gran Sasso, 67010 Assergi, L'Aquila, Italy \\ ${ }^{3}$ Institut Laue-Langevin, 71 Avenue des Martyrs, 38000 Grenoble, France \\ ${ }^{4}$ B. P. Konstantinov Petersburg Nuclear Physics Institute of National Research Centre 'Kurchatov Institute', 188300 Gatchina, Leningrad Region, \\ Russia
}

Received: 23 March 2018 / Accepted: 19 August 2018 / Published online: 5 September 2018

(C) The Author(s) 2018

\begin{abstract}
Present experimental and astrophysical limits do not exclude that the neutron $(n)$ oscillation into mirror neutron $\left(n^{\prime}\right)$, a sterile state exactly degenerate in mass with the neutron, can be a very fast process, in fact faster than the neutron decay itself, in which case it would have very interesting implications in cosmology and astrophysics. This process is sensitive to the magnetic field. Namely, if the mirror magnetic field $\mathbf{B}^{\prime}$ exists at the Earth, $n-n^{\prime}$ oscillation probability can be suppressed or resonantly amplified by the applied magnetic field $\mathbf{B}$, depending on its strength and on the angle $\beta$ between $\mathbf{B}$ and $\mathbf{B}^{\prime}$. We present the results of ultra-cold neutron storage measurements aiming to check the anomalies observed in previous experiments which could be a signal for $n-n^{\prime}$ oscillation in the presence of mirror magnetic field $B^{\prime} \sim 0.1 \mathrm{G}$. From the analysis of the experimental data new lower limits on $n-n^{\prime}$ oscillation time as a function of $B^{\prime}$ were obtained, assuming that the mirror magnetic field is constant in time: $\tau_{n n^{\prime}}>17 \mathrm{~s}\left(95 \%\right.$ C.L.) for any $B^{\prime}$ between 0.08 and $0.17 \mathrm{G}$, and $\tau_{n n^{\prime}} / \sqrt{\cos \beta}>27 \mathrm{~s}\left(95 \%\right.$ C.L.) for any $B^{\prime}$ in the interval $(0.06 \div 0.25) \mathrm{G}$.
\end{abstract}

\section{Introduction}

The existence of mirror particles was proposed by Lee and Yang, in the same paper where the possibility of parity violation was put forward [1], for restoring parity in more extended sense: for our particles being left-handed, Parity can be interpreted as a discrete symmetry which exchanges them with their right-handed mirror partners. Hence the parity, violated in each of ordinary and mirror sectors separately, would remain as an exact symmetry between two sectors. Kobzarev,

a e-mail: berezhiani@lngs.infn.it
Okun and Pomeranchuk [2] observed that mirror particles cannot have ordinary strong, weak or electromagnetic interactions, and so they must form a hidden parallel world as an exact duplicate of ordinary one interacting with normal matter via gravity. This idea was further expanded, with different twists, in many subsequent papers [3-25]. (See reviews [2628]; for a historical overview, see also Ref. [29]).

At the basic level, one can consider a theory based on a direct product $G \times G^{\prime}$ of identical gauge factors which can naturally emerge e.g. in the $E_{8} \times E_{8}^{\prime}$ string theory. Ordinary particles belong to the Standard Model $G=$ $S U(3) \times S U(2) \times U(1)$ or its grand unified extension, while the gauge interactions $G^{\prime}=S U(3)^{\prime} \times S U(2)^{\prime} \times U(1)^{\prime}$ (or its respective extension) describes mirror particles. The total Lagrangian must have a form $\mathcal{L}_{\text {tot }}=\mathcal{L}+\mathcal{L}^{\prime}+\mathcal{L}_{\text {mix }}$ where the Lagrangians $\mathcal{L}$ and $\mathcal{L}^{\prime}$, which describe the particle interactions respectively in observable and mirror sectors, can be rendered identical by imposing a mirror parity $G \leftrightarrow G^{\prime}$ exchanging ordinary and mirror fermions modulo their chirality. Thus, if mirror sector exists, then all our particles: the electron $e$, proton $p$, neutron $n$, photon $\gamma$, neutrinos $v$ etc. must have invisible mass degenerate mirror twins: $e^{\prime}, p^{\prime}, n^{\prime}$, $\gamma^{\prime}, v^{\prime}$ etc. which are sterile to our strong and electroweak interactions but interact with ordinary particles via universal gravity.

Mirror matter can be a viable candidate for dark matter [19-28]. The possible interactions between the particles of two sectors (encoded in $\mathcal{L}_{\text {mix }}$ ), as the kinetic mixing between photon and mirror photon [9-13] or interactions mediated by heavy messengers coupled to both sectors, as gauge bosons/gauginos of common flavor symmetry [30] or common $B-L$ symmetry [31,32], can induce mixing phenomena between ordinary and mirror particles. In fact, any 
neutral particle, elementary or composite, might have a mixing with its mirror twin. E.g. the photon kinetic mixing [9-13] can be searched experimentally via the positronium - mirror positronium oscillation [33-37] and also via direct detection of dark matter [38-40]. The gauge bosons of common flavor symmetry [30] can induce the mixing between the neutral pions and Kaons and their mirror partners, also with implications for dark matter direct search [41]. Three ordinary neutrinos $v_{e, \mu \tau}$ can oscillate into their (sterile) mirror partners $v_{e, \mu \tau}^{\prime}[14-18]$. The respective mass-mixing terms can emerge via the effective interactions which violate $B-L$ symmetries of both sectors. These interactions can be induced via the seesaw mechanism by heavy gauge singlet "right-handed" neutrinos [23-25] which interact with both ordinary and mirror leptons. On the other hand, the same $B-L$ non-conserving interactions would induce $\mathrm{CP}$ violating processes between ordinary and mirror particles and thus generate the baryon asymmetries in both sectors [23-25]. In this way, the relation between the dark and observable matter fractions in the Universe, $\Omega_{B}^{\prime} / \Omega_{B} \simeq 5$, can be naturally explained [23-28].

As it was shown in Refs. [42,43], the present probes do not exclude the possibility that oscillation between the neutron $n$ and its mirror twin $n^{\prime}$ is a rather fast process, in fact faster than the neutron decay. The mass mixing, $\varepsilon\left(\bar{n} n^{\prime}+\bar{n}^{\prime} n\right)$, emerges from $B$-violating six-fermion effective operators of the type $(u d d)\left(u^{\prime} d^{\prime} d^{\prime}\right) / M^{5}$ involving ordinary $u, d$ and mirror $u^{\prime}, d^{\prime}$ quarks, with $M$ being a cutoff scale related to new physics beyond the Fermi scale. As far as the masses of $n$ and $n^{\prime}$ are exactly equal, they must have maximal mixing in vacuum and oscillate with timescale $\tau_{n n^{\prime}}=\varepsilon^{-1} \sim(M / 10 \mathrm{TeV})^{5} \mathrm{~s}$. Existing experimental limits or cosmological/astrophysical bounds cannot exclude oscillation time $\tau_{n n^{\prime}}=\tau$ of few seconds [42]. ${ }^{1}$ It is of key importance that in nuclei $n \rightarrow n^{\prime}$ transition is forbidden by energy conservation and thus nuclear stability bounds give no limitations on $\tau$, while for free neutrons $n-n^{\prime}$ oscillation is affected by magnetic fields and coherent interactions with matter which makes this phenomenon rather elusive $[42,43]$. On the other hand, it is striking that $n \rightarrow n^{\prime}$ transitions faster than the neutron decay can have far going implications for the propagation of ultra-high energy cosmic rays at cosmological distances [47-49], for the neutrons from solar flares [50], for primordial nucleosynthesis [51] and for neutron stars [25]. ${ }^{2}$

\footnotetext{
${ }^{1}$ For comparison, the neutron-antineutron oscillation time is restricted to be larger than few years by the direct experimental limit $\tau_{n \bar{n}}>0.86 \times$ $10^{8} \mathrm{~s}$ [44] as well as the indirect limits from the stability of nuclei, see Refs. $[45,46]$ for a recent review.

2 In principle, $n-n^{\prime}$ transition can occur not only due to mass mixing term $\varepsilon \bar{n} n^{\prime}+$ h.c. , but also due to transitional magnetic moment $\delta \mu\left(F_{\mu \nu}+F_{\mu \nu}^{\prime}\right) \bar{n} \sigma^{\mu v} n^{\prime}+$ h.c. between $n$ and $n^{\prime}$ states $[53,54]$. However, we do not discuss this possibility here and concentrate only on the mass-mixing effects.
}

The possibility of fast $n-n^{\prime}$ oscillations can be tested in experiments searching for neutron disappearance $n \rightarrow n^{\prime}$ and regeneration $n \rightarrow n^{\prime} \rightarrow n$ [42] as well as via non-linear effects on the neutron spin precession [43]. In the ultra-cold neutron (UCN) traps $n \rightarrow n^{\prime}$ conversion can be manifested via the magnetic field dependence of the neutron loss rates. For the UCN flight times between wall collisions $t \sim 0.1 \mathrm{~s}$, the experimental sensitivity can reach $\tau \sim 500 \mathrm{~s}$ [55] (see also Ref. [56] for a recent status of the UCN sources for fundamental physics measurements).

Several experiments searched for $n-n^{\prime}$ oscillation with the UCN traps [57-61]. Following the naive assumption [42] that the Earth has no mirror magnetic field, these experiments compared the UCN loss rates in zero (i.e. small enough) and non-zero (large enough) magnetic fields. In this case the probability of $n-n^{\prime}$ oscillation after a time $t$ depends on the applied magnetic field $B$ as $P_{B}(t)=\sin ^{2}(\omega t) /(\omega \tau)^{2}, \omega=$ $\frac{1}{2}|\mu \mathbf{B}|=(B / 1 \mathrm{mG}) \times 4.5 \mathrm{~s}^{-1}$, where $\mu=-6 \cdot 10^{-12} \mathrm{eV} / \mathrm{G}$ is the neutron magnetic moment. ${ }^{3}$ For small fields $(B<1$ $\mathrm{mG}$ or so, when $\omega t<1)$ one has $P_{0}=(t / \tau)^{2}$, while for large fields $(B>20 \mathrm{mG}$ or so, when $\omega t \gg 1)$ oscillations are suppressed, $P_{B}<(1 / \tau \omega)^{2} \ll(t / \tau)^{2}$. In this way, lower bounds on the oscillation time were obtained under the no mirror field hypothesis, the strongest being $\tau>414 \mathrm{~s}$ at $90 \%$ CL [58] adopted by the Particle Data Group [62].

However, the above limits become invalid in the presence of mirror matter and/or mirror magnetic field [43]. If the Earth possesses mirror magnetic field $B^{\prime}$, then it would show up as uncontrollable background suppressing $n-n^{\prime}$ oscillation even if the ordinary magnetic field is screened in the experiments, i.e. $B=0$. However, if experimental magnetic field is tuned as $B \approx B^{\prime}$, then $n-n^{\prime}$ oscillation would be resonantly amplified. In addition, in this case one could observe the strong dependence of the UCN losses on the direction of magnetic field [43].

Interestingly, some of the experiments have shown that the UCN loss rates depend on the magnetic field direction at certain values of magnetic fields, in particular, the measurements performed with vertical magnetic fields $B \simeq 0.2 \mathrm{G}$ reported in Ref. [60]. The detailed analysis of these experimental data indicates towards $5.2 \sigma$ deviation from the null hypothesis [63] which can be interpreted as a signal for $n-n^{\prime}$ oscillation in the presence of mirror magnetic field $B^{\prime} \sim 0.1 \mathrm{G}$ at the Earth.

A dedicated experiment [61] tested $n-n^{\prime}$ oscillation in the presence of mirror magnetic field, with a series of measurements at different values of applied (vertical) magnetic field varied from 0 to $0.125 \mathrm{G}$ with a step of $0.025 \mathrm{G}$, also altering its direction from up to down. Its results, yielding the limit $\tau>12 \mathrm{~s}$ for any $B^{\prime}$ less than $0.13 \mathrm{G}$, restrict the parameter

\footnotetext{
${ }^{3}$ Hereafter we use natural units, $\hbar=c=1$.
} 
space $\left(\tau, B^{\prime}, \beta\right)$ which can be responsible for the above $5.2 \sigma$ anomaly but do not cover it completely.

In this paper we report the results of additional measurements aiming to test the parameter space related to $5.2 \sigma$ anomaly [63]. We essentially repeated the experiment [60] with different values of the applied magnetic field. New limits on $n-n^{\prime}$ oscillation time were obtained as a function of mirror magnetic field $B^{\prime}$, which strongly restrict the parameter space relevant for the above anomaly, however still leave some margins for it. The paper is organized as follows. First we discuss $n-n^{\prime}$ oscillation in the presence of mirror magnetic field. Then we describe the experiment and show its results. At the end we confront our findings with the results of previous experiments and draw our conclusions.

\section{Oscillation $n-n^{\prime}$ in the presence of mirror magnetic fields}

The hypothesis that the Earth might possess a mirror magnetic field, with the strength comparable to the Earth ordinary magnetic field, might be a not too exotic possibility. The Earth may capture some amount of mirror matter [25] if there exist strong enough interactions between ordinary and mirror particles, e.g. due to photon-mirror photon kinetic mixing. In fact, geophysical data on the Earth mass, moment of inertia, normal mode frequencies etc. still allow the presence of dark matter in the Earth with a mass fraction up to $4 \times 10^{-3}$ [64]. Due to a high temperature in the Earth core, the captured mirror matter might be ionized, at least partially. Then the drag of free mirror electrons by the Earth rotation, induced by their Rutherford-like scatterings off ordinary matter, again due to the photon kinetic mixing, could give rise to circular mirror currents inducing the mirror magnetic field. Such a mechanism of the electron drag was proposed in Ref. [65] and applied to the generation of the galactic magnetic fields. The dynamo effects could additionally enforce the mirror magnetic field at the Earth and also change its configuration, so that it could also exhibit significant variations in time $[25,43]$.

For free neutrons propagating in the vacuum but in the presence of ordinary $B$ and mirror $B^{\prime}$ magnetic fields which are both non-zero and arbitrarily oriented, $n-n^{\prime}$ oscillation is described by the Schrödinger equation with a $4 \times 4$ Hamiltonian:

$i \frac{d \Psi}{d t}=H \Psi, \quad H=\left(\begin{array}{cc}\mu B \sigma & \varepsilon \\ \varepsilon & \mu B^{\prime} \sigma\end{array}\right)$

where $\Psi(t)=\left(\psi_{n}(t), \psi_{n^{\prime}}(t)\right)$ is is the wave function of $n$ and $n^{\prime}$ each containing two $( \pm)$ spin states, $\psi_{n}=\left(\psi_{n}^{+}, \psi_{n}^{-}\right)$ and $\psi_{n^{\prime}}=\left(\psi_{n^{\prime}}^{+}, \psi_{n^{\prime}}^{-}\right)$, and $\sigma=\left(\sigma_{x}, \sigma_{y}, \sigma_{z}\right)$ are the Pauli matrices. The exact calculation of $n-n^{\prime}$ oscillation probability is given in Ref. [43]. In homogeneous fields $\mathbf{B}$ and $\mathbf{B}^{\prime}$, the probability of $n \rightarrow n^{\prime}$ transition after a time $t$ can be conveniently reduced to the formula given in Ref. [63]:

$$
\begin{aligned}
P_{\mathbf{B B}^{\prime}}(t) & =\mathcal{P}_{B B^{\prime}}(t)+\mathcal{D}_{\mathbf{B B}^{\prime}}(t) \\
& =\mathcal{P}_{B B^{\prime}}(t)+D_{B B^{\prime}}(t) \cos \beta,
\end{aligned}
$$

where $\beta$ is the angle between the vectors $B$ and $B^{\prime}$ and

$\mathcal{P}_{B B^{\prime}}(t)=\frac{\sin ^{2}\left[\left(\omega-\omega^{\prime}\right) t\right]}{2 \tau^{2}\left(\omega-\omega^{\prime}\right)^{2}}+\frac{\sin ^{2}\left[\left(\omega+\omega^{\prime}\right) t\right]}{2 \tau^{2}\left(\omega+\omega^{\prime}\right)^{2}}$,
$D_{B B^{\prime}}(t)=\frac{\sin ^{2}\left[\left(\omega-\omega^{\prime}\right) t\right]}{2 \tau^{2}\left(\omega-\omega^{\prime}\right)^{2}}-\frac{\sin ^{2}\left[\left(\omega+\omega^{\prime}\right) t\right]}{2 \tau^{2}\left(\omega+\omega^{\prime}\right)^{2}}$,

where $\tau=\varepsilon^{-1}, \omega=\frac{1}{2}|\mu B|$ and $\omega^{\prime}=\frac{1}{2}\left|\mu B^{\prime}\right|$. Hence, for given values of $\omega$ and $\omega^{\prime}$ the oscillation amplitude (2) becomes maximal or minimal respectively when $\mathbf{B}$ and $\mathbf{B}^{\prime}$ are parallel or anti-parallel, $\cos \beta= \pm 1$ :

$P_{B B^{\prime}}^{(+)}(t)=\mathcal{P}_{B B^{\prime}}(t)+D_{B B^{\prime}}(t)=\frac{\sin ^{2}\left[\left(\omega-\omega^{\prime}\right) t\right]}{\tau^{2}\left(\omega-\omega^{\prime}\right)^{2}}$,
$P_{B B^{\prime}}^{(-)}(t)=\mathcal{P}_{B B^{\prime}}(t)-D_{B B^{\prime}}(t)=\frac{\sin ^{2}\left[\left(\omega+\omega^{\prime}\right) t\right]}{\tau^{2}\left(\omega+\omega^{\prime}\right)^{2}}$

Hence, using these expressions, the oscillation probability (2) can be presented also as

$P_{\mathbf{B B}^{\prime}}(t)=P_{B B^{\prime}}^{(+)}(t) \cos ^{2} \frac{\beta}{2}+P_{B B^{\prime}}^{(-)}(t) \sin ^{2} \frac{\beta}{2}$.

In the experiments one cannot control the mirror magnetic field. However, ordinary magnetic field can be varied and thus the dependence of $n-n^{\prime}$ conversion probability on $B$ can be detected. In particular, by reversing the magnetic field direction $B \rightarrow-B$ (i.e. $\beta \rightarrow \pi-\beta$ ) the probability (2) becomes $P_{-\mathbf{B B}^{\prime}}=\mathcal{P}_{B B^{\prime}}-D_{B B^{\prime}} \cos \beta$. Therefore, $P_{\mathbf{B B}^{\prime}}-P_{-\mathbf{B B}^{\prime}}=2 D_{B B^{\prime}} \cos \beta$ is non-zero unless $\cos \beta=0$. On the other hand, the sum of probabilities $P_{\mathbf{B B}^{\prime}}+P_{-\mathbf{B B}^{\prime}}=$ $\mathcal{P}_{B B^{\prime}}$ does not depend on the angle $\beta$, and it can be compared with the oscillation probability in zero magnetic field, $P_{\mathbf{0 B}}{ }^{\prime}=\mathcal{P}_{0 B^{\prime}}$. In particular, if the mirror magnetic field is large enough, so that $\omega^{\prime} t>1$, which for flight times $t \sim 0.1 \mathrm{~s}$ implies $B^{\prime}>1 \mathrm{mG}$ or so, the averaged oscillation probability is $\mathcal{P}_{0 B^{\prime}}=\frac{1}{2}\left(\omega^{\prime} \tau\right)^{-2}$. On the other hand, at $B \approx B^{\prime}$, when $\left|\omega-\omega^{\prime}\right| t<1$, oscillation probability will resonantly amplify, $\mathcal{P}_{B B^{\prime}} \approx \frac{1}{2}(t / \tau)^{2} \gg \mathcal{P}_{0 B^{\prime}}$.

When the neutrons are stored in the UCN traps, the oscillation probability (2) should be averaged over the distribution of neutron flight times $t$ between the wall collisions. For averaging sinusoidal factors in (4) one can use the analytic formula suggested in Ref. [63]:

$\left\langle\sin ^{2}(\omega t)\right\rangle_{t}=S(\omega)=\frac{1}{2}\left[1-e^{-2 \omega^{2} \sigma_{\mathrm{f}}^{2}} \cos \left(2 \omega t_{\mathrm{f}}\right)\right]$,

where $t_{\mathrm{f}}=\langle t\rangle$ is the neutron mean free-flight time between wall collisions and $\sigma_{\mathrm{f}}^{2}=\left\langle t^{2}\right\rangle-t_{\mathrm{f}}^{2}$. For an UCN trap of given 
geometrical form and sizes these characteristic times can be computed via Monte Carlo simulations, and typically one has $t_{\mathrm{f}}, \sigma_{\mathrm{f}} \sim 0.1 \mathrm{~s}$. Eq. (6) gives a correct asymptotic behavior for the average probabilities (4):

$\bar{P}_{B B^{\prime}}^{( \pm)}=\frac{S\left(\omega \mp \omega^{\prime}\right)}{\tau^{2}\left(\omega \mp \omega^{\prime}\right)^{2}}$

In the limit $\left|\omega-\omega^{\prime}\right| t_{\mathrm{f}} \gg 1$ the second term in Eq. (6) is negligible and one can set $S\left(\omega \pm \omega^{\prime}\right)=1 / 2$, which is equivalent to averaging of $\sin ^{2}$ factors in (4). So we get

$\overline{\mathcal{P}}_{B B^{\prime}}=\frac{\omega^{2}+\omega^{\prime 2}}{2 \tau^{2}\left(\omega^{2}-\omega^{\prime 2}\right)^{2}}, \quad \bar{D}_{B B^{\prime}}=\frac{\omega \omega^{\prime}}{\tau^{2}\left(\omega^{2}-\omega^{\prime 2}\right)^{2}}$.

Explicit form (6) of $S\left(\omega-\omega^{\prime}\right)$ is relevant close to the resonance, when $\left|\omega-\omega^{\prime}\right| t_{\mathrm{f}} \ll 1$, or $\left|B-B^{\prime}\right|<1 \mathrm{mG}$. Then one gets $S\left(\omega-\omega^{\prime}\right) \approx\left(\omega-\omega^{\prime}\right)^{2}\left\langle t^{2}\right\rangle$ and thus $\bar{P}_{B B^{\prime}}^{(+)} \approx\left\langle t^{2}\right\rangle / 2 \tau^{2}$. As we show below, in traps with the homogeneous magnetic field the mean probabilities calculated with the analytic approximation (6) agree very well (about a per cent accuracy) to that obtained via Monte-Carlo simulations.

Oscillation $n-n^{\prime}$ can be tested via magnetic field dependence of UCN losses. In the absence of $n-n^{\prime}$ conversion the number of neutrons $N\left(t_{*}\right)$ survived after effective storage time $t_{*}$ in the trap from the initial amount should not depend on $\mathbf{B}$, as far as the usual UCN losses during the storage as are the neutron decay, wall absorption or upscattering are magnetic field independent in the standard physics framework. However, if a neutron between the wall collisions oscillates into a sterile state $n^{\prime}$, then per each collision the latter can escape from the trap. Hence, the amount of survived neutrons in the UCN trap with applied magnetic field $B$ after a time $t_{*}$ is given by $N_{\mathbf{B}}\left(t_{*}\right)=N\left(t_{*}\right) \exp \left(-n_{*} \bar{P}_{\mathbf{B B}}\right)$, where $N\left(t_{*}\right)$ is a "would be" amount of survived UCN in the absence of $n-n^{\prime}$ oscillation, $\bar{P}_{\mathbf{B B}^{\prime}}$ is the average probability of $n-n^{\prime}$ conversion between the wall scatterings and $n_{*}=n\left(t_{*}\right)$ is the mean number of wall scatterings for the neutrons survived after the time $t_{*}$. If the magnetic field direction is inverted, $B \rightarrow-B$, then the amount of survived neutrons would become $N_{-\mathbf{B}}\left(t_{*}\right)=N\left(t_{*}\right) \exp \left(-n_{*} \bar{P}_{-\mathbf{B B}^{\prime}}\right)$. Since the common factor $N\left(t_{*}\right)$ cancels in the neutron count ratios, asymmetry between $N_{\mathbf{B}}\left(t_{*}\right)$ and $N_{-\mathbf{B}}\left(t_{*}\right)$,

$A_{\mathbf{B}}\left(t_{*}\right)=\frac{N_{-\mathbf{B}}\left(t_{*}\right)-N_{\mathbf{B}}\left(t_{*}\right)}{N_{-\mathbf{B}}\left(t_{*}\right)+N_{\mathbf{B}}\left(t_{*}\right)}$,

should directly trace the difference $\bar{P}_{\mathbf{B B}^{\prime}}-\bar{P}_{-\mathbf{B B}^{\prime}}=\overline{\mathcal{D}}_{\mathbf{B B}^{\prime}}$ [43]. Assuming $n_{*} \overline{\mathcal{D}}_{\mathbf{B B}} \ll 1$, we get

$A_{\mathbf{B}}\left(t_{*}\right) / n_{*}=\overline{\mathcal{D}}_{\mathbf{B B}^{\prime}}=\bar{D}_{B B^{\prime}} \cos \beta$
On the other hand, one can compare the average $N_{B}\left(t_{*}\right)=$ $\frac{1}{2}\left[N_{\mathbf{B}}\left(t_{*}\right)+N_{-\mathbf{B}}\left(t_{*}\right)\right]$ with the counts $N_{0}\left(t_{*}\right)$ acquired under zero magnetic field:

$E_{B}\left(t_{*}\right)=\frac{N_{0}\left(t_{*}\right)-N_{B}\left(t_{*}\right)}{N_{0}\left(t_{*}\right)+N_{B}\left(t_{*}\right)}$

This value measures the difference between the probabilities in zero and non-zero magnetic fields. Since $\bar{P}_{\mathbf{B B}^{\prime}}+\bar{P}_{-\mathbf{B B}^{\prime}}=$ $2 \overline{\mathcal{P}}_{B B^{\prime}}$, we have

$E_{B}\left(t_{*}\right) / n_{*}=\overline{\mathcal{P}}_{B B^{\prime}}-\overline{\mathcal{P}}_{0 B^{\prime}}=\bar{\Delta}_{B B^{\prime}}$,

which should not depend on the magnetic orientation but only on its modulus $B=|\mathbf{B}|$, and it can be resonantly amplified if $B \approx B^{\prime}$. Therefore, measuring $E_{B}$ at different values of $B$, one can obtain direct limits on $n-n^{\prime}$ oscillation time $\tau$, while by measuring $A_{\mathbf{B}}$ one in fact measures the value $\tau_{\beta}=\tau / \sqrt{|\cos \beta|}$, i.e. is the oscillation time corrected for the unknown angle $\beta$ between ordinary and mirror magnetic fields $B$ and $B^{\prime}$. Once again, in ideal conditions these measurements should have no systematic uncertainties: measuring the neutron counts in different magnetic configurations but otherwise in the same experimental conditions, the effects of the regular UCN losses should cancel in the count ratios $A_{\mathbf{B}}$ and $E_{B}$, and scanning over different test values of applied magnetic field $\mathbf{B}$ with appropriate statistics, one can obtain pretty stringent limits on $\tau$ and $\tau_{\beta}$ as a function of mirror magnetic field $B^{\prime}$.

\section{Experiment and measurements}

Our experiment was carried out at the Research Reactor of the Institute Laue-Langevin (ILL), Grenoble, using the EDM beam-line of the UCN facility PF2. The vacuum chamber of PNPI spectrometer was used, the same that in previous experiments on $n-n^{\prime}$ transitions $[58,60]$. The experimental set up consisting of a neutron guiding system, the UCN storage trap with valves for filling and emptying, two UCN detectors D1 and D2, and magnetic shielding is shown in Fig. 1. The trap of $190 \ell$ volume capable of storing about half million UCN has a form of cylinder with a length $120 \mathrm{~cm}$ and diameter $45 \mathrm{~cm}$. Its inner surface is coated by beryllium. The trap is located inside a shield which screens the Earth magnetic field (for more details, see Refs. [58,60]).

A controlled magnetic field $B$ was applied inside the trap using electric circuits placed on the top and bottom of the chamber (red contours in Fig. 1). For a given electric current in the circuits, the value of the induced magnetic field and its direction at each position inside the trap were calculated theoretically, by approximating the interior of the trap by a 


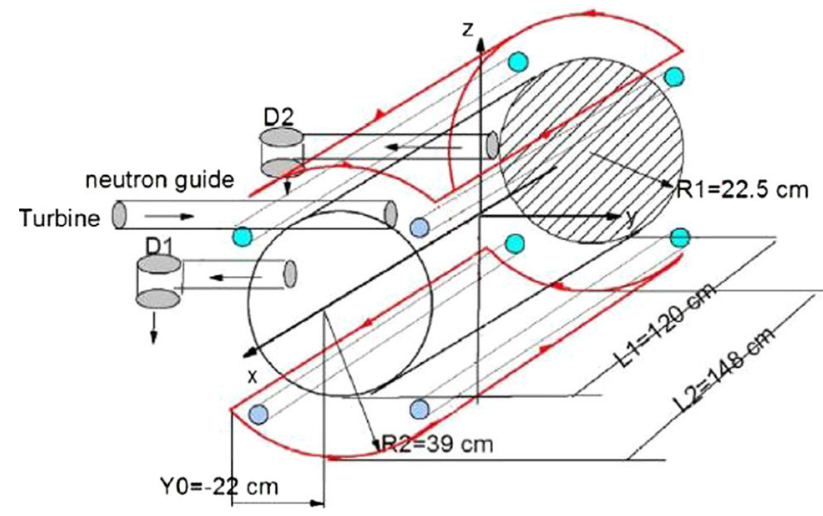

Fig. 1 Scheme of the UCN chamber used in the experiment. Positions of entrance valve from the neutron guide and exit valves to detectors D1 and D2 are shown. Electric circuits inducing magnetic field in the trap are shown by red contours

cubic lattice, with coordinates $x, y, z$ from the center of the trap taken with $1 \mathrm{~cm}$ steps. The obtained results were also checked with a magnetometer at center and some characteristic lateral parts of the chamber. The induced magnetic field B had practically vertical direction everywhere but its magnitude $B=|\mathbf{B}|$ had wide distribution varying from the value $B_{c}$ in the center by about $\pm 0.5 B_{c}$ at peripheral regions. The distribution of magnetic field inside the trap is shown in Fig. 2. In the following, for describing different experimental configurations we shall use the central value of magnetic field $B_{c}$ induced by a proper electric current. Direction of magnetic field was periodically inverted by changing the direction of current.

The scheme of the experiment is the following. Each measurement consists of five phases: monitoring, filling, storage, emptying and background fixing. Typical time per measurement including the turbine waiting time is about $10 \mathrm{~min}$. The monitoring phase is used to check the stability of the UCN flux from the reactor. After the entrance valve is open, neutrons flow into the trap via the UCN guide while the exit valves towards two detectors D1 and D2 remain open, and their counts during monitoring time $t_{\mathrm{m}}=50 \mathrm{~s}$ are used as estimators of the incident UCN flux. Then the exit valves are closed for a filling time of $100 \mathrm{~s}$, after which the entrance valve is closed and the $\mathrm{UCN}$ are confined inside the trap for a holding time $t_{\mathrm{s}}=250 \mathrm{~s}$. Then the exit valves are reopen and the survived UCN are counted by two detectors during the emptying time of $150 \mathrm{~s}$. The background phase is for checking that no excess of neutrons remain inside the trap that could influence the subsequent measurement.

In first three series of experiments $(B 1, B 2, B 3)$ the asymmetry $A_{\mathbf{B}}$ (9) was measured employing only large magnetic fields $\left(B_{c}=0.21 \mathrm{G}, B_{c}=0.12 \mathrm{G}\right.$ and $B_{c}=0.09 \mathrm{G}$ respectively), repeating the cycles $\{B\}=$ $\{-B,+B,+B,-B ;+B,-B,-B,+B\}$ with the $\mathrm{UCN}$ holding time $t_{\mathrm{s}}=250 \mathrm{~s}$ (signs \pm correspond to the
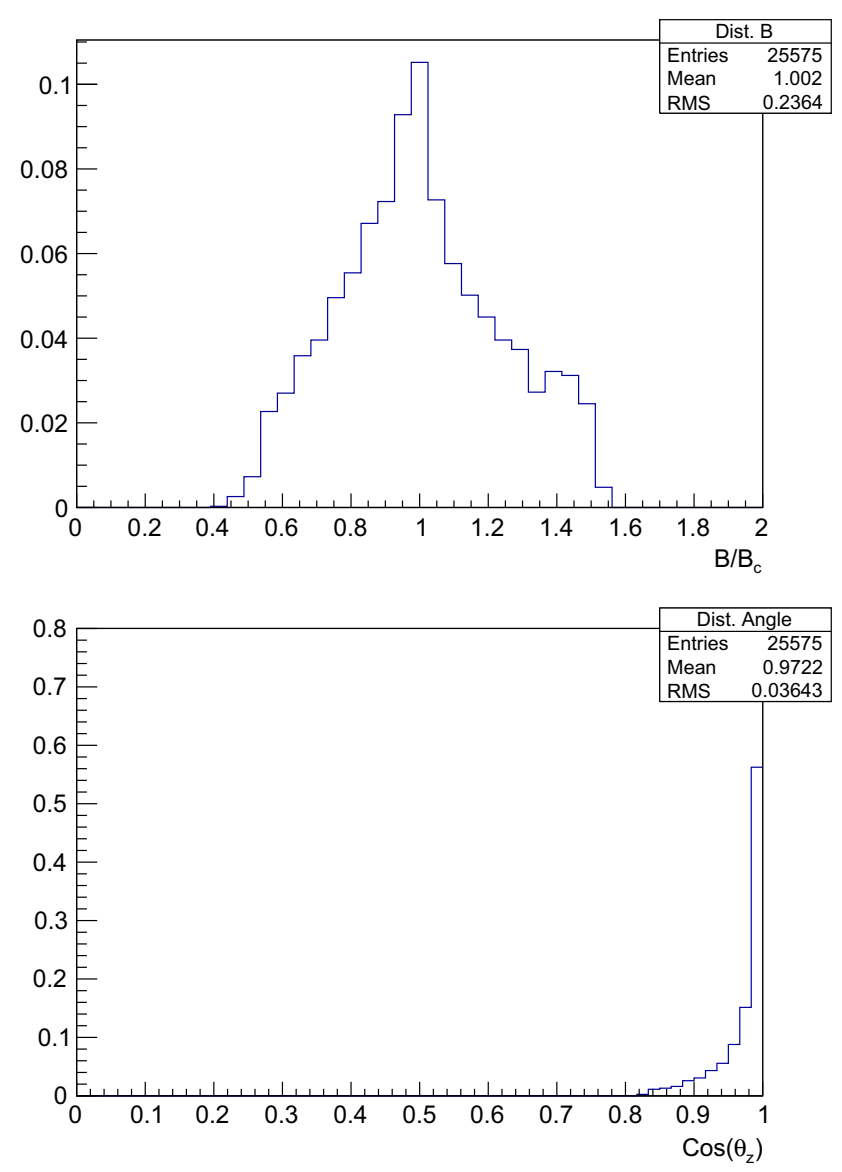

Fig. 2 Upper panel: distribution of magnetic field around the central value $B_{c}$ inside the trap. Lower panel: distribution of $\left|\cos \theta_{z}\right|$ values where $\theta_{z}$ is the angle between vector $\mathbf{B}$ and vertical $z$ axis

fields directed up and down). In last part of series $B 4$, again with $B_{c}=0.12 \mathrm{G}$, we used the $\{B\}$-mode measurements only for initial period and then switched to the measurement sequences $\{0 \mid B\}=\{0,+B,-\mathbf{B}, \mathbf{0} ; 0,-\mathbf{B},+B, 0\}$, altering zero and non-zero values of magnetic field. For technical reasons, in all series $B 4$ only one detector (D1) was used and the holding time was reduced to $t_{\mathrm{s}}=150 \mathrm{~s}$. Time gap between the series $B 3$ and $B 4$ was devoted to calibration measurements for testing possible systematic effects that could render the detector counts sensitive to the magnetic field strength and its orientation, as e.g. influence of the alternating current on the counting electronics. These measurements were performed with high statistics in continuous flow regime for $\{B\}$ mode at $B_{c}=0.09 \mathrm{G}$ and $B_{c}=0.12 \mathrm{G}$, with entrance and both exit valves of the trap open during $200 \mathrm{~s}$ so that the neutrons entering the trap were finishing in one of two detectors after a short diffusion time. Asymmetries $A_{\mathbf{B}}$ of neutron counts $N_{\mathbf{B}}$ and $N_{-\mathbf{B}}$ acquired in this regime by both detectors D1 and D2 were compatible with zero, making it clear that the switching of magnetic fields had no influence. After series $B 4$, the similar calibration measurements were performed 
also in $\{0 \mid B\}$ mode with $B_{c}=0.12 \mathrm{G}$ and $B_{c}=0.21 \mathrm{G}$, using only detector $\mathrm{D} 1$.

The averaged number of wall collisions $n_{*}$ and mean probabilities of $n-n^{\prime}$ oscillation (3) between collisions were estimated via a Monte Carlo (MC) simulation. It consisted of two steps which we briefly describe here. (The detailed description is given in Ref. [66].)

First we estimated the average number of wall scatterings taking into account that the initial velocity spectrum of the UCN [67] entering the trap gradually degrades during the storage time due to regular neutron losses. Therefore, a MC simulation was performed, first without considering the effects of $n-n^{\prime}$ oscillation, in order to obtain the mean value of free flight time $t_{\mathrm{f}}=\langle t\rangle$, its variance $\left\langle t^{2}\right\rangle$, etc. In this way, the distribution of above values were computed by averaging them over the individual neutron trajectories in the trap, using well-known formulas [68] for the UCN losses per scattering. The fit parameters which were the slope of the initial (Maxwellian) spectrum of the neutrons and the UCN loss factor $\eta$, were adjusted for reproducing the experimental data as characteristic time constants for the neutron counts during the phases of monitoring, trap filling, UCN storage, and the trap emptying. The obtained values are in good agreement with the parameters used in the previous experiments with the same trap $[58,60]$. In this way, for the obtained fir parameters, for a given storage time $t_{\mathrm{s}}$ we computed an average amount of wall scatterings $n_{*}$ that survived neutrons suffered starting from the moment they enter the trap in the stage of filling, to the moment when they hit the detectors in the emptying phase. $^{4}$

Namely, we get $n_{*}=2068 \pm 18$ for $t_{\mathrm{s}}=250 \mathrm{~s}$ in $B 1, B 2, B 3$ modes and $n_{*}=1487 \pm 15$ for $t_{\mathrm{s}}=150 \mathrm{~s}$ in $B 4$ mode with one detector. The above error-bars related to fitting uncertainties introduce about $1 \%$ systematic errors in the determination of $n-n^{\prime}$ oscillation time. In the following, for deducing our limits in more conservative way, we use the lower values of $n_{*}$.

At second step, we computed average oscillation probabilities (2) over the neutron flight time. Given that the applied magnetic field in our experiment was not homogeneous, the empirical formula (6) cannot be used: for given central value $B_{c}$ in the trap, the neutrons during their diffusion can cross resonant values $B=B^{\prime}$ with some probability if the value of mirror magnetic field falls inside the distribution profile of the experimental magnetic field in the trap . Therefore, for each experimental series, we calculated the oscillation probability between wall scatterings as a function of $B^{\prime}$ fol-

\footnotetext{
${ }^{4}$ Since $n-n^{\prime}$ oscillation can take place not only during the UCN holding time $t_{\mathrm{s}}$ but also during filling and emptying of the trap, the effective exposure time can be estimated as $t_{*}=t_{\mathrm{s}}+70 \mathrm{~s}$, as in previous experiments with the same trap $[58,60]$. Let us remark that for us the relevant parameter, rather than $t_{*}$, is $n_{*}$ computed directly via the MC simulation.
}
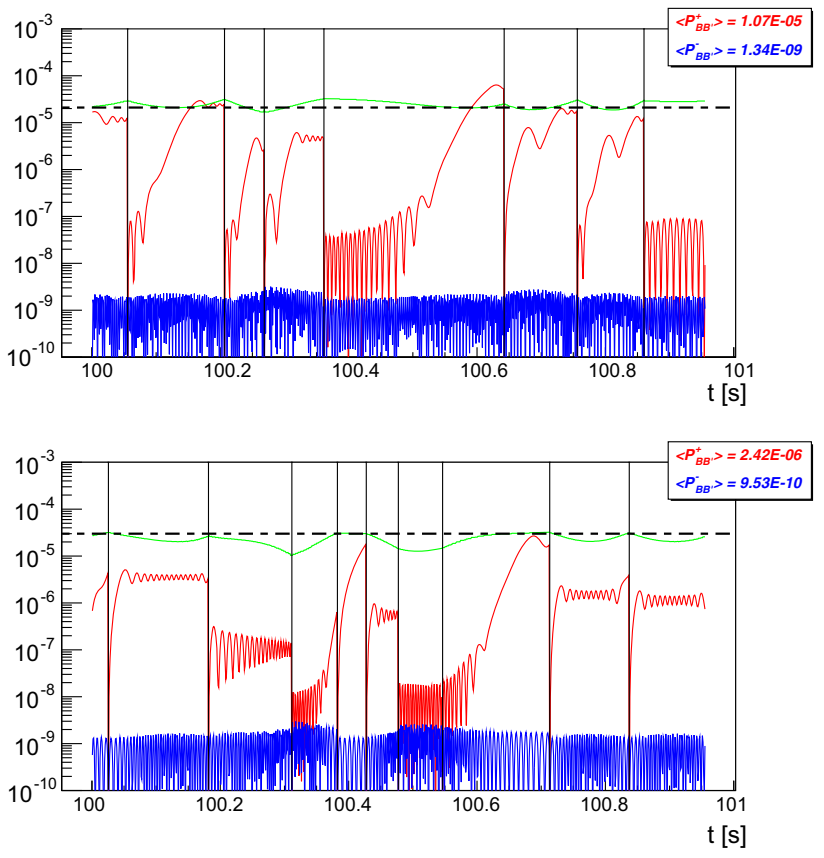

Fig. 3 Examples of time evolution of $n-n^{\prime}$ oscillation probabilities $P_{B B^{\prime}}^{ \pm}(t)$ (4) between wall scatterings for an UCN with $v=4 \mathrm{~m} / \mathrm{s}$. For definiteness, the central field is taken as $B_{c}=0.21 \mathrm{G}$ and $n-n^{\prime}$ oscillation time is set as $\tau=10 \mathrm{~s}$. Mirror field (shown by horizontal dash lines in Tesla units) is taken as $B^{\prime}=0.2 \mathrm{G}$ (upper panel) or $B^{\prime}=0.3 \mathrm{G}$ (lower panel). Green curves show the profile of applied magnetic field (also in Tesla) which the neutron crossed during its diffusion in the trap. Red and blue curves correspond to $P_{B B^{\prime}}^{+}(\beta=0)$ and $P_{B B^{\prime}}^{-}(\beta=\pi)$. At each wall scattering (marked by black vertical lines) the wave function resets to the pure neutron state. In insets we show the corresponding average probabilities per wall scattering

lowing the neutron trajectories in the trap. The interior of the trap was represented by a cubic lattice with $1 \mathrm{~cm}^{3}$ elementary volumes, the magnetic field $\mathbf{B}$ was calculated in every node of this lattice, and in this way the distributions shown in Fig. 2 were obtained. Inside each elementary $\mathrm{cm}^{3}$ volume the magnetic field was taken as a constant value obtained by averaging between 8 calculated values at its vertices. The Schrödinger equation (1) was numerically integrated along the neutron trajectories. Every neutron leaving an elementary cube with a given value of magnetic field with a wave-vector $\Psi=\left(\psi_{n}, \psi_{n^{\prime}}\right)$, while crossing the adjacent cube was evolving with the another magnetic field value corresponding to this adjacent cube. At each wall scattering the wave vector was reset to the pure state of neutron $\Psi=(1,0)$. The typical evolution of $n-n^{\prime}$ oscillation probabilities $P_{B B^{\prime}}^{( \pm)}(t)(4)$ for a $1 \mathrm{~s}$ period of the neutron diffusion in the trap is shown in Fig. 3, where in insets we show the average value of this probabilities per wall scattering during this period.

In this way, via MC simulations we computed mean values $\bar{P}_{B B^{\prime}}^{( \pm)}$of the probabilities (4) between wall scatterings, averaged over distribution of the neutron flight time $t$ and distribution of the magnetic field $B$ in the trap for a given 

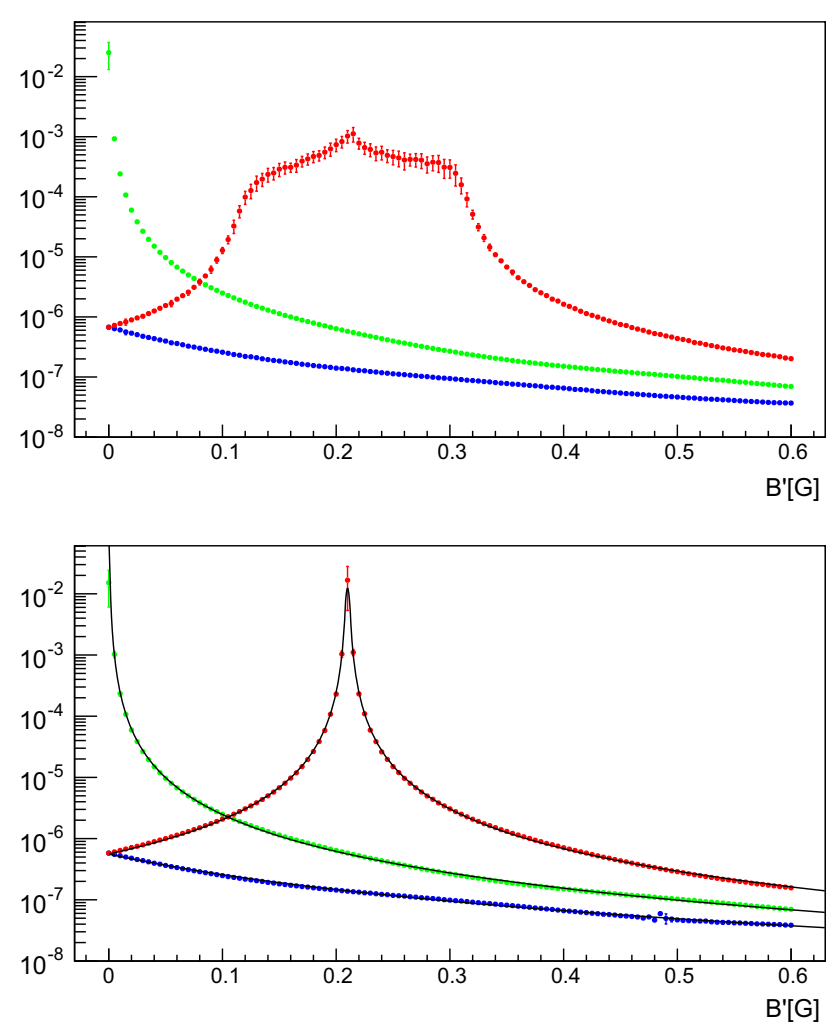

Fig. 4 Upper panel: Red and blue points show results of MC simulation respectively for $S_{+}\left(B^{\prime}\right)$ and $S_{-}\left(B^{\prime}\right)$ in our trap with $B_{c}=0.21 \mathrm{G}$, and green points results for $S_{0}\left(B^{\prime}\right)$ (when magnetic field in trap is vanishing, $B=0$ ). Lower panel: The results of the same MC simulations the case of homogeneous magnetic field $B=0.21 \mathrm{G}$ and $B=0$. Black solid curves show the results obtained via empirical formula (6), in perfect coincidence with the $\mathrm{MC}$ results

value of $B_{c}$. In addition, we computed also the mean oscillation probability $\bar{P}_{0 B^{\prime}}$ for the case when no magnetic field was applied, $B=0$.

$\bar{P}_{B B^{\prime}}^{( \pm)}=\left\langle\frac{\sin ^{2}\left[\left(\omega \mp \omega^{\prime}\right) t\right]}{\tau^{2}\left(\omega \mp \omega^{\prime}\right)^{2}}\right\rangle_{t, B}=\left(\frac{1 \mathrm{~s}}{\tau}\right)^{2} S_{ \pm}\left(B^{\prime}\right)$,

$\bar{P}_{0 B^{\prime}}=\left\langle\frac{\sin ^{2}\left(\omega^{\prime}\right) t}{\tau^{2} \omega^{\prime 2}}\right\rangle_{t}=\left(\frac{1 \mathrm{~s}}{\tau}\right)^{2} S_{0}\left(B^{\prime}\right)$.

In upper panel of Fig. 4 we show $S_{ \pm}\left(B^{\prime}\right)$ and $S_{0}\left(B^{\prime}\right)$ as functions of mirror field strength $B^{\prime}$. As we see, these factors correspond to mean values of the respective probabilities normalized to $n-n^{\prime}$ oscillation time $\tau=1 \mathrm{~s}$.

For checking the consistency of our MC simulation, we also computed average oscillation probabilities (13) in the case of homogeneous magnetic field in the same trap (lower panel of Fig. 4). As we see, the results of MC simulation perfectly coincide with the results (black solid curves) obtained via the empirical formula (6) with the corresponding MC values of $t_{\mathrm{f}}=\langle t\rangle$ and $\sigma_{\mathrm{f}}^{2}=\left\langle t^{2}\right\rangle-t_{\mathrm{f}}^{2}$. As we see from Fig. 4 , wide profile of magnetic field distribution as in Fig. 2 has certain advantages: while the function $S_{+}\left(B^{\prime}\right)$ in homoge- neous magnetic field has larger "peak" sensitivity close to the resonance $B^{\prime} \approx B_{c}$ than in inhomogeneous field, in the latter case it is sensitive to much wider range of $B^{\prime}$.

\section{Data analysis}

Different datasets $B 1, B 2, B 3$ and $B 4$ were independently analyzed and the values of asymmetries $A_{\mathbf{B}}(9)$ were computed via comparing the neutron counts $N_{\mathbf{B}}$ and $N_{-\mathbf{B}}$ between subsequent measurements. For each individual measurement under given applied field $\mathbf{B}$ we take $N_{\mathbf{B}}$ as a sum of counts of both detectors, $N_{\mathbf{B}}=N_{\mathbf{B}}^{(1)}+N_{\mathbf{B}}^{(2)}$, while the count ratios between two detectors $N_{\mathbf{B}}^{(1)} / N_{\mathbf{B}}^{(2)}$ were also controlled as the stability check. Assuming Poisson statistics, the errors can be estimated as $\Delta N_{\mathbf{B}}=\sqrt{N_{\mathbf{B}}}$. For eliminating the effects of drift, in our analysis we use the values of $A_{\mathbf{B}}$ averaged within the measurement octets $\{B\}$ for series $B 1, B 2, B 3$ and $\{0 \mid B\}$ for series $B 4$. Hence, a cycle $\{B\}$ of 8 measurements yield an average of 4 measured values for $A_{\mathbf{B}}$ while 8 measurements of cycle $\{0 \mid B\}$ yield 2 values of $A_{\mathbf{B}}$ and 2 values of $E_{B}$. In this way, for series $B 4$ also the values $E_{B}$ (11) were computed. In addition, the UCN counts $M_{\mathbf{B}}$ and $M_{-\mathbf{B}}$ in the monitoring phase were also controlled, and detector-to-monitor normalized asymmetries $A_{B}^{\text {nor }}$ between the ratios $(N / M)_{\mathbf{B}}$ and $(N / M)_{-\mathbf{B}}$, and analogously $E_{B}^{\text {nor }}$. In this way, the average values of $A_{\mathbf{B}}$ and $E_{B}$ obtained in each measurement series, were transformed into mean probabilities $\overline{\mathcal{D}}_{\mathbf{B}}=A_{\mathbf{B}} / n_{*}$ (10) and $\bar{\Delta}_{B}=E_{B} / n_{*}$ (12) taking the mean amount of wall scatterings computed via MC simulations as $n_{*}=2050$ for configurations $B 1, B 2, B 3$ and $n_{*}=1472$ for $B 4$.

The results obtained per each measurement series are shown in Table 1 and also on Fig. 5 where the measured asymmetries are combined in bins of comparable size. The first column of Table 1 indicates the values measured in a given series, and corresponding amount $N_{\text {oct }}$ of the measurement octets $\{B\}$ or $\{0 \mid B\}$. Let us remind that for canceling the effects of drift, each data unit is taken as value of $A_{\mathbf{B}}$ or $E_{B}$ averaged within a given octet of measurements. Thus for a constant fit the amount of degrees of freedom per each series is $N_{\text {oct }}-1$. The second column of Table 1 shows $\overline{\mathcal{D}}_{\mathbf{B}}$ and $\bar{\Delta}_{B}$ deduced respectively from the average values of $A_{\mathbf{B}}$ and $E_{B}$ in each series, and the expected statistical errors (with statistical fluctuation for every count $N$ taken as $\sqrt{N}$ ). However, the corresponding values of $\chi^{2} /$ d.o.f. (in parenthesis) are too large which indicates that these fits are not that good and experimental results have larger dispersion than one expects from statistics Third column shows the mean values of $A_{\mathbf{B}}$ and $E_{B}$ and respective variances obtained directly from the distribution of their measured values in each series. As we see, the central values in third column are consistent to that of second column, however the error bars are larger. In fact, 
Table 1 Results for $\overline{\mathcal{D}}_{\mathbf{B}}$ and $\bar{\Delta}_{B}$ obtained from the average values of $A_{B}$ and $E_{B}$ measured in respective experimental cycles (in square brackets the amount of measurement octets in each cycle) taking into account only statistical errors (in parenthesis the quality of constant fit $\left(\chi^{2} /\right.$ d.o.f. $)$ is shown $)$. Last column shows the mean values and variance reconstructed directly from the distribution of the experimental results

\begin{tabular}{lrr}
\hline Series $\left[N_{\text {oct }}\right]$ & \multicolumn{1}{c}{ Stat. $\left[\times 10^{-8}\right]$} & Dist. $\left[\times 10^{-8}\right]$ \\
\hline$A_{B 1} / n_{*}[74]$ & $-1.59 \pm 5.40(1.57)$ & $-1.12 \pm 7.09$ \\
$A_{B 1}^{\text {nor }} / n_{*}[74]$ & $0.43 \pm 5.89(1.73)$ & $0.99 \pm 7.67$ \\
$A_{B 2} / n_{*}[124]$ & $-14.9 \pm 3.80(2.90)$ & $-14.9 \pm 6.40$ \\
$A_{B 2}^{\text {nor }} / n_{*}[124]$ & $-16.6 \pm 4.14(2.84)$ & $-16.6 \pm 6.70$ \\
$A_{B 3 / n_{*}}[57]$ & $-0.03 \pm 5.79(1.92)$ & $-1.54 \pm 8.39$ \\
$A_{B 3}^{\text {nor }} / n_{*}[57]$ & $1.93 \pm 6.32(1.83)$ & $0.96 \pm 9.07$ \\
$A_{B 4} / n_{*}[43]$ & $4.18 \pm 7.47(2.20)$ & $4.57 \pm 12.1$ \\
$A_{B 4}^{\text {nor }} / n_{*}[43]$ & $8.61 \pm 9.28(2.50)$ & $8.67 \pm 14.3$ \\
$E_{B 4} / n_{*}[28]$ & $13.0 \pm 13.0(2.20)$ & $12.8 \pm 20.4$ \\
$E_{B 4}^{\text {nor }} / n_{*}[28]$ & $13.7 \pm 13.7(1.94)$ & $13.7 \pm 22.4$ \\
\hline
\end{tabular}

the latter errors well coincide with the respective statistical errors enlarged by the respective value of $\sqrt{\chi^{2} / \text { d.o.f. }}$

Wide black crosses in Fig. 5 show the mean values of $A_{\mathbf{B}} / n_{*}$ and $A_{\mathbf{B}}^{\text {nor }} / n_{*}$ and respective errors per each series obtained directly from the dispersion of the measured values, in correspondence to third column of Table 1. The dashed black crosses show the same for $E_{B} / n_{*}$ and $E_{B}^{\text {nor }} / n_{*}$, and grey crosses show results of calibration measurements. Shaded squares show mean values per each bin and statistical errors, while the larger error-bars indicate the data dispersion in each bin.

In ideal situation, our measurements should have no systematical uncertainties since the regular neutron losses should not affect the values of $A_{\mathbf{B}}$ or $E_{B}$ measured in the same experimental conditions. Hence, in the absence of $n-n^{\prime}$ oscillations one expects that the values $A_{\mathbf{B}}$ and $E_{B}$ should be consistent with zero within statistical errors. Table 1 shows that average values measured in each experimental series are consistent with null hypothesis within $1 \sigma$ statistical errors except that of largest series $B 2$, comprising over 200 hours of continuous measurements, where the values $A_{\mathbf{B}}$ and $A_{\mathbf{B}}^{\text {nor }}$ both show about $4 \sigma$ deviation from zero. On the other hand, the quality of constant fits presented in Table 1 is not that good (namely, $\chi^{2} /$ d.o.f. $=2.9$ for series $B 2$ ) which means that some unaccounted external factors were influencing our measurements.

As one can see on Fig. 5, the results of series $B 2$ (and to some extent also of series $B 1$ ) show a strong dispersion between different bins which should be a main reason for bad constant fit. (In contrast, the results of series $B 3$ show no significant dispersion between different bins.) Second column of Table 1 for series $B 2$ shows almost $4 \sigma$ deviation from nullhypothesis if only statistical errors are taken into account,
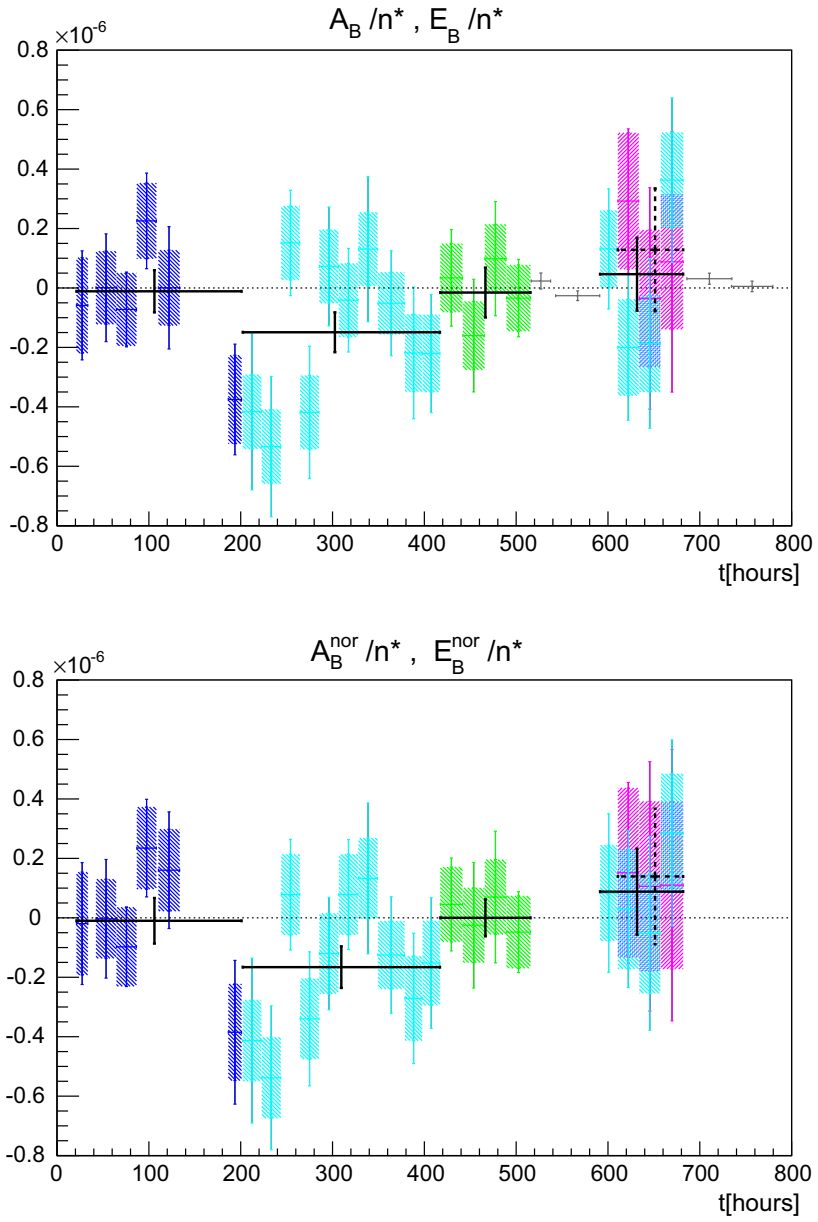

Fig. 5 Binned results for $\overline{\mathcal{D}}_{\mathbf{B}}$ (upper panel) and $\overline{\mathcal{D}}_{\mathbf{B}}^{\text {nor }}$ (lower panel) from the data acquired in configurations $B 1\left(B_{c}=0.21 \mathrm{G}\right.$, blue $), B 2$ $\left(B_{c}=0.12 \mathrm{G}\right.$, cyan $), B 3\left(B_{c}=0.09 \mathrm{G}\right.$, green $)$ and $B 4\left(B_{c}=0.12 \mathrm{G}\right.$, again cyan). Shaded squares correspond to expected statistical errors, while the data dispersion in bins are indicated by longer error bars. Magenta squares and error bars indicate the results for $\bar{\Delta}_{B}$ and $\bar{\Delta}_{B}^{\text {nor }}$ from $\{0 \mid B\}$-mode measurements of series $B 4$. Grey crosses in upper panel correspond to calibration series

namely the values $A_{B 2} / n_{*}$ and $A_{B 2}^{\text {nor }} / n_{*}$ are deviated from zero respectively by 3.9 and $4.0 \sigma$. On the other hand, even with enlarged error bars (third column of Table 1) both values $A_{\mathbf{B}}$ and $A_{\mathbf{B}}^{\text {nor }}$ of series $B 2$ still have respectively 2.3 and $2.5 \sigma$ deviations from zero. The same result can be obtained by averaging between the bins of series $B 2$ with enlarged error bars which shows that this discrepancy is pretty robust against the methods of the analysis. In principle, this situation could be interpreted as a signal of $n-n^{\prime}$ oscillation in the presence of mirror magnetic field $B^{\prime} \sim 0.1 \div 0.2 \mathrm{G}$ with a direction varying in time. However, duration and acquired statistics of our experiment is not enough to draw such a far going conclusion. Therefore, we take more conservative attitude, and in the following we use the mean values and their variances obtained directly from the distribution of the measured values of $A_{\mathbf{B}}$ and $E_{B}$ per each series, shown in third column 
of Table 1. In addition, we average between the results of series $B 2$ and $B 4$ performed under the same magnetic field $B_{c}=0.12 \mathrm{G}$ and thus obtain

$\overline{\mathcal{D}}_{\mathbf{B B}^{\prime}}\left[B_{c}=0.12 \mathrm{G}\right]=(-10.4 \pm 5.70) \times 10^{-8}$,

$\overline{\mathcal{D}}_{\mathbf{B B}^{\prime}}^{\mathrm{nor}}\left[B_{c}=0.12 \mathrm{G}\right]=(-11.8 \pm 6.10) \times 10^{-8}$.

This averages have less than $2 \sigma$ deviation from zero and thus can be used for setting $95 \%$ C.L. on the oscillation time $\tau_{\beta}$ as a function of mirror magnetic field $B^{\prime}$ assuming that the vector $\mathbf{B}^{\prime}$ was constant in time.

\section{Results for $n-n^{\prime}$ oscillation parameters}

Experimental values of $E_{B} / n_{*}=\bar{\Delta}_{B B^{\prime}}$ and $A_{\mathbf{B}} / n_{*}=$ $\overline{\mathcal{D}}_{\mathbf{B B}^{\prime}}=\bar{D}_{B B^{\prime}} \cos \beta$ shown in Table 1 can be transformed into the $n-n^{\prime}$ oscillation parameters $\tau^{2}$ and $\tau^{2} / \cos \beta$ via Eq. (13):

$$
\begin{aligned}
& \frac{1}{\tau^{2}}\left[\mathrm{~s}^{-2}\right]=\bar{\Delta}_{B B^{\prime}}^{\exp }\left[\frac{S_{+}\left(B^{\prime}\right)+S_{-}\left(B^{\prime}\right)}{2}-S_{0}\left(B^{\prime}\right)\right]^{-1} \\
& \frac{\cos \beta}{\tau^{2}}\left[\mathrm{~s}^{-2}\right]=\overline{\mathcal{D}}_{B B^{\prime}}^{\exp }\left[S_{+}\left(B^{\prime}\right)-S_{-}\left(B^{\prime}\right)\right]^{-1} .
\end{aligned}
$$

The obtained results are shown in Fig. 6. Dash magenta curve shows values of $1 / \tau^{2}$ as function of $B^{\prime}$ reproduced from central values of $E_{B} / n_{*}$ in Table 1 , while solid magenta curve corresponds to $95 \%$ C.L upper limit on $1 / \tau^{2}$ obtained via taking into account respective error-bars of third column. Dash cyan curve shows central values of $\cos \beta / \tau^{2}$ obtained from central value of $\overline{\mathcal{D}}_{B B^{\prime}}$ in (14), an average result between the measurements $B 2$ and $B 4$ with about $2 \sigma$ deviation from zero, while solid cyan contours confine corresponding $95 \%$ C.L. area. (let us remind that $\cos \beta$ can be positive or negative; here $\beta=0$ corresponds to mirror magnetic field directed to the Earth center.) Blue and green solid contours show $95 \%$

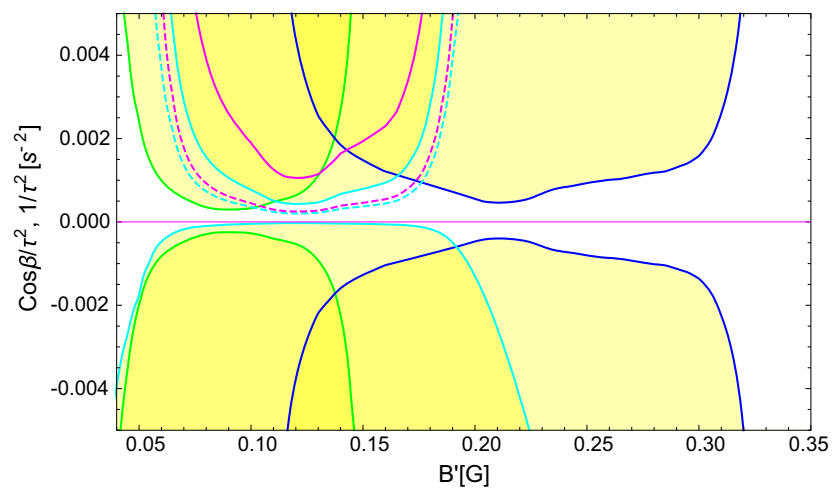

Fig. 6 Exclusion regions for $1 / \tau^{2}$ and $\cos \beta / \tau^{2}$ extracted from our measurements of $E_{B}$ and $A_{B}$. Curves of different colors, corresponding to the colors of bins in Fig. 5 confine regions excluded by measurements at different values of $B_{c}$, respectively for positive and negative $\cos \beta$ in upper and lower parts of this Figure
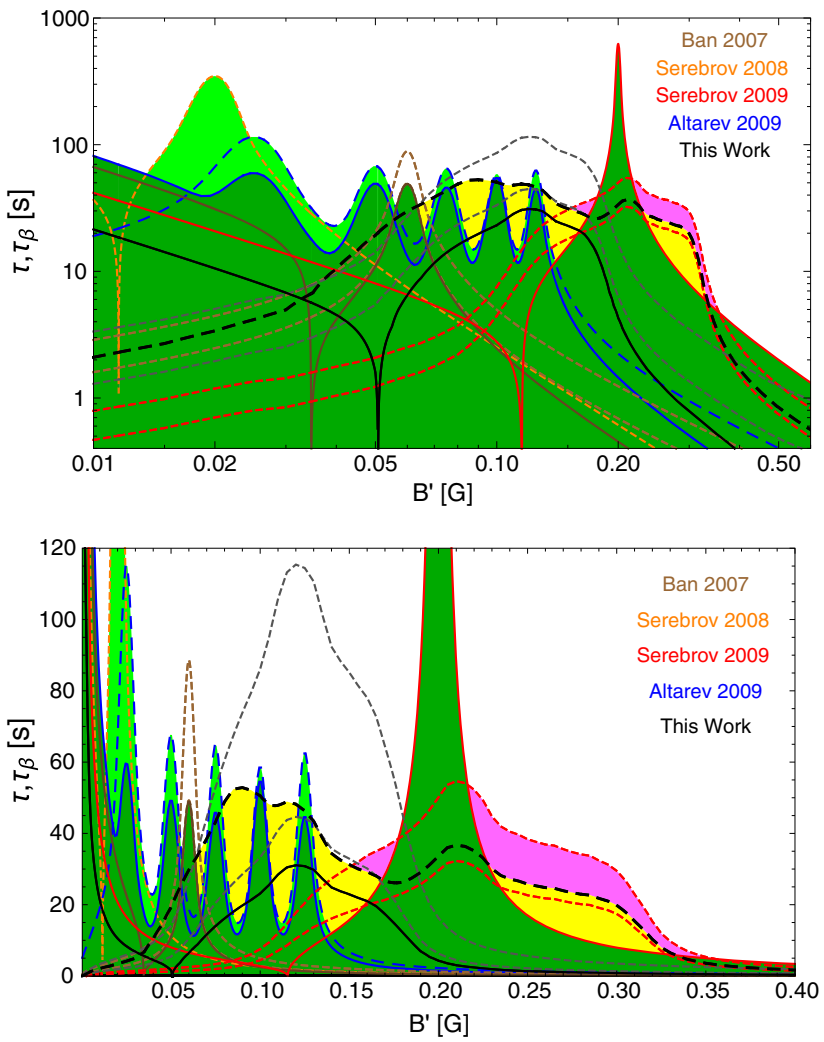

Fig. 7 upper panel: our lower limits on $\tau$ (black solid) and $\tau_{\beta}$ (black dashed). The parameter areas excluded by the previous experiments are shaded in dark green for $\tau$ and light green for $\tau_{\beta}$ while the yellow shaded area corresponds to new regions excluded in this work (all exclusion limits correspond to $95 \%$ C.L.). Solid red curve corresponds to lower limit on $\tau$ from experiment Serebrov et al. [60] while dashed red contours confine $2 \sigma$ region of $\tau_{\beta}$ relevant for $5.2 \sigma$ anomaly in vertical magnetic fields with $B_{c}=0.2 \mathrm{G}[60,63]$. The wavy blue curves show lower limits on $\tau$ (solid) and $\tau_{\beta}$ (dashed) from the results of Altarev et al. [61]. Orange dashed contour corresponds to the limit from Serebrov [58]. Solid brown curve corresponds to lower limit on $\tau$ from experiment Ban et al. [57] while the dotted brown curves confine $2 \sigma$ region for $\tau_{\beta}$ relevant for $3 \sigma$ deviation of $A_{\mathbf{B}}$ in the same experiment. The parameter areas relevant for these anomalous deviations that are not excluded are shaded in pink. Dashed grey contours confine the $2 \sigma$ parameter region corresponding to $2.5 \sigma$ deviation in our measurements of $B 2$ series. Lower panel: the same in linear scale, as the blow up of the exclusion regions covered by our experiment and previous experiments

C.L. limits on $\cos \beta / \tau^{2}$ deduced from results for $A_{B} / n_{*}$ for series $B 1$ and $B 3$, third column of Table 1 .

In Fig. 7 we show results of global fit of our experimental data, $95 \%$ C.L. lower limits on $\tau$ (black solid) and $\tau_{\beta}$ (black dashed), and confront them with the results of previous experiments. The parameter areas excluded by previous experiments are shaded in green and additional areas excluded by our experiment are shaded in yellow.

In particular, the first experiment Ban et al. [57] searching for $n-n^{\prime}$ oscillation compared the UCN losses between measurements in zero magnetic field $B=0$ and homogeneous non-zero field $B=0.06 \mathrm{G}$ while the direction of the 
latter was altered between vertical up $(+\mathbf{B})$ and down $(-\mathbf{B})$. No significant deviation from zero was found in the value of $E_{B}$ (11). The corresponding $95 \%$ C.L. lower limit on $\tau$ as a function of $B^{\prime}$ is shown by solid brown curve in Fig. 7. On the other hand, the data reported in Table 1 of Ref. [57], indicate towards a non-zero asymmetry between the counts $N_{\mathbf{B}}$ and $N_{-\mathbf{B}}$ in all measurement series with different storage times (see also Table 1 of Ref. [43]). In overall, this corresponds to $3 \sigma$ deviation of $A_{\mathbf{B}}(9)$ from zero which can be interpreted via $n-n^{\prime}$ oscillation in the presence of mirror magnetic field Ref. [43]. The $2 \sigma$ allowed area corresponding to this deviation is shown by dash brown contours.

The second experiment Serebrov et al. [58] used zero magnetic field $B=0$ and homogeneous non-zero field $B=0.02 \mathrm{G}$ directed horizontally. It obtained strongest limit under vanishing mirror field assumption, $\tau>414 \mathrm{~s}$ at 90 $\%$ C.L. (or $\tau>386 \mathrm{~s}$ at $95 \%$ C.L.). Since the direction of non-zero field was not altered in this experiment, one cannot obtain direct limits on $\tau$ and $\tau_{\beta}$ for $B^{\prime} \neq 0$. Nevertheless, in Fig. 7 we show the indicative parameter area (confined by dashed orange curve) which would be excluded at $95 \%$ C.L. under assumption that mirror field had a strictly vertical direction, independently up or down.

The next experiment Serebrov et al. [60] performed measurements in $\{\mathbf{0} \mid \mathbf{B}\}$ mode using horizontally directed homogeneous magnetic field $B=0.2 \mathrm{G}$. The $95 \%$ C.L. lower limit on $\tau$ as a function of $B^{\prime}$ resulting from these measurements is shown by red solid curve in Fig. 7. However, in the same experiment Serebrov et al. [60], the part of measurements were performed also in in $\{\mathbf{B}\}$ mode with vertical magnetic field with central value $B_{c}=0.2 \mathrm{G}$, which has shown substantial asymmetry between the counts $N_{\mathbf{B}}$ and $N_{-\mathbf{B}}$. The detailed analysis of these experimental data performed in Ref. [63] for the asymmetry $A_{\mathbf{B}}$ indicate towards 5.2 $\sigma$ deviation from the null hypothesis, which can be interpreted as a signal of $n-n^{\prime}$ oscillation in the background of mirror magnetic field $B^{\prime} \simeq 0.1 \div 0.2 \mathrm{G}$. Let us remark that in Ref. [63] the consequences of this anomaly for $\tau_{\beta}$ as a function of $B^{\prime}$ was deduced assuming the homogeneity of the applied magnetic field and using the profile of functions $S_{ \pm}\left(B^{\prime}\right)$ (13) calculated via the analytic formula (6), as shown on lower panel of Fig. 4. However, as we realized while performing our experiment in the same conditions, the magnetic field distribution was rather wide, and so the parameter area corresponding to the observed 5.2 $\sigma$ deviation as reproduced in Ref. [63] was not quite correct. Therefore, as far as the experiment Serebrov et al. [60] and our experiment were performed using the same UCN chamber, in the present paper we recalculated $\tau_{\beta}$ as a function of $B^{\prime}$ using properly the functions $S_{ \pm}\left(B^{\prime}\right)$ computed via our MC simulations (upper panel of Fig. 4). The red dashed contours in Fig. 7 confine the obtained $2 \sigma$ area corresponding to this anomaly.
The experiment Altarev et al. [61] was performed to search $n-n^{\prime}$ oscillation in the presence of mirror magnetic field, by varying the values of applied magnetic field (vertical) from 0 to $0.125 \mathrm{G}$ with a step of $0.025 \mathrm{G}$ and also altering its direction from up to down. In Fig. 7 we show $95 \%$ C.L. lower limits on $\tau$ (blue solid) and $\tau_{\beta}$ (blue dashed) as functions of $B^{\prime}$ obtained via fitting the data reported in Fig. 1 or Ref. [61]. Overally, these limits exclude $\tau<12 \mathrm{~s}$ and $\tau_{\beta}<15 \mathrm{~s}$ at $95 \%$ C.L. for any $B^{\prime}$ in the interval from $(0 \div 0.13) \mathrm{G}$. For mirror fields smaller than $0.01 \mathrm{G}$, the lower limits on $\tau$ or $\tau_{\beta}$ are stronger, $\tau>80 \mathrm{~s}$ at $95 \%$ C.L. for $B^{\prime}=0.01 \mathrm{G}$ and approaching $\tau>386 \mathrm{~s}$ for $B^{\prime}=0$, as obtained in Ref. [63] by combining the results of Ref. [61] with that of Ref. [58]. The limits that can be obtained from the results of Ref. [59] are much weaker and completely covered by above discussed limits, so we do not show them here.

In Fig. 7 parameter areas excluded by the previous experiments in overall are shaded in dark green for $\tau$ and light green for $\tau_{\beta}=\tau / \sqrt{\cos \beta}$ while the yellow shaded areas correspond to new regions excluded in our work. Namely, solid and dashed black contours confine the $95 \%$ C.L. excluded areas respectively for $\tau$ and $\tau_{\beta}$. Short dashed grey contours limit $2 \sigma$ area corresponding to $2.5 \sigma$ deviation in $A_{B 2}^{\text {nor }} / n_{*}$ in our measurements of series $B 2$, see Table 1 , which actually corresponds to $4 \sigma$ deviation at level of statistical errors but we treated it more conservatively due to large $\chi^{2} /$ d.o.f. The pink shaded areas correspond to parameter regions relevant for $5.2 \sigma$ anomaly of Refs. $[60,63]$ which still remain allowed by the present experimental limits. As we see from Fig. 7, this latter anomaly measured with $B_{c}=0.2 \mathrm{G}$ is compatible with our $2.5 \sigma$ deviation in measurements of series $B 2$ with $B_{c}=0.12 \mathrm{G}$ (see the region between two grey dashed curves) for a small parameter area around $B^{\prime}=0.16 \mathrm{G}$ and $\tau_{\beta}=30 \mathrm{~s}$ corresponding to the left part of the pink shaded region in Fig. 7).

\section{Conclusions and outlook}

The aim of this experiment was to test the results of experiment Serebrov et al. [60] with vertical magnetic fields at $B_{c}=0.2 \mathrm{G}$, showing $5.2 \sigma$ deviation from the null hypothesis [63] that could be interpreted as a signal for $n-n^{\prime}$ oscillation in the presence mirror magnetic field (see the parameter area confined between two red dashed curves in Fig. 7 which in fact implies an upper limit $\tau \leq \tau_{\beta}<57$ s at $95 \%$ C.L.). The results of other experiments restrict the parameter space relevant for this anomaly but cannot exclude it completely. In particular, lower limits on $\tau$ obtained from the same experiment [60] with horizontal and homogeneous magnetic field $B=0.2 \mathrm{G}$ (solid red contour in Fig. 7) are compatible with the above anomaly for the range $B^{\prime}=(0.08 \div 0.35) \mathrm{G}$ with respective values of $\tau$ ranging from $5 \mathrm{~s}$ to $55 \mathrm{~s}$. Results of 
Ref. [61] (blue solid and dashed contours in Figs. 7) yield the lower limits $\tau>12 \mathrm{~s}$ and $\tau_{\beta}>15 \mathrm{~s}$ for any $B^{\prime}$ less than $0.13 \mathrm{G}$. Combined with the above bounds of Ref. [60] with "horizontal" measurements, the limit $\tau_{\beta} \geq \tau>12 \mathrm{~s}$ can be extended got the range of mirror fields up to $B^{\prime}=0.25 \mathrm{G}$.

Our experimental results enhance these experimental limits, and also for a wider range of possible values of $B^{\prime}$ (see yellow shaded regions in Fig. 7). Namely, for any $B^{\prime}$ in the interval $(0.08 \div 0.17) \mathrm{G}$ we get a lower limit on $n-n^{\prime}$ oscillation time $\tau>17 \mathrm{~s}$ (95\% C.L.), and $\tau_{\beta}>27 \mathrm{~s}$ for any $B^{\prime}$ in the interval $(0.06 \div 0.25)$ G. Assuming that the mirror magnetic field $B^{\prime}$ is constant in time, or in more precise terms, that its value and direction did not change significantly in time during the years passed from previous experiments to our measurements, we can combine our results with limits of previous works. Yet, we could not completely exclude the parameter areas of interest, and pink shaded regions in Fig. 7 correspond to regions which still can be relevant for above mentioned 5.2 $\sigma$ anomaly. (Interestingly, the left part of the pink shaded area, with $B^{\prime}=(0.15 \div 0.17) \mathrm{G}$ and $\tau_{\beta}=(28 \div 35) \mathrm{s}$, is also compatible with $2.5 \sigma$ deviation observed in $B 2$ series of our measurements.) For larger values of $B^{\prime}$ the limit on $\tau$ and $\tau_{\beta}$ is considerably weakened and for $B>0.35 \mathrm{G}$ the values of $n-n^{\prime}$ oscillation time as small as few seconds remains allowed.

The following remark is in order. The results of different experiments performed in different times can be combined only if one assumes that mirror magnetic field is constant in time. However, this is the most naive assumption, which means that rotations of the Earth and the Baby "mirror Earth" in its interior are completely synchronous, so that the orientation of the mirror magnetic field in the given experimental site does not change in time. On the other hand, if the axis of mirror dipole is deviated from its rotation axis, and there is some difference between angular velocities by which ordinary and magnetic fields precess, then the periodic variation of the signal can be expected. In addition, the captured mirror matter in the deep interior of the Earth can come into thermal equilibrium with the normal matter and thus can be present in the ionized form, due to a temperature of the Earth core of several thousand $\mathrm{K}$. This can cause other periodic effects related to the Moon tides on the mirror gas inside the Earth. In addition, the dynamo mechanism in the differentially rotating mirror plasma could play a substantial role, mirror magnetic field can strongly increase and also its configuration can change from dipole to multipolar or toroidal configuration, with the subsequent inversion of the direction of the mirror dipole. In this the mirror magnetic field of the Earth may have also substantial large period time variation, perhaps of few years, like the sun's magnetic field. and it can be increased up to several Gauss. Unfortunately, time duration of our experiment (one reactor cycle) is not enough to place limits on possible long period time variation of mirror magnetic field. However, the possibility of time varying mirror field background should be taken in consideration while planning the next experiments searching for $n-n^{\prime}$ oscillation, as e.g. $n \rightarrow n^{\prime} \rightarrow n$ regeneration experiment with cold neutrons at the stage of preparation at HFIR Reactor of the Oak Ridge national Laboratory $[69,70]$ or any future experiments with the UCN storage chambers.

Acknowledgements We are grateful to the Institut Laue-Langevin (ILL) for providing the EDM beamline on PF2 facility for one reactor cycle and for excellent technical support throughout the experimental runs. The work was supported in part by CETEMPs at the University of L'Aquila. Z.B. and R.B. acknowledge the partial financial support from the ILL. We are extremely grateful to Anatoly Serebrov for extraordinary help in resolving various questions and valuable advices. Z.B. thanks Yuri Kamyshkov, Valery Nesvizhevsky and Guido Visconti for interest to the work and useful discussions, and brothers Fillip Pozzi, Michel Pozzi, M.E.Pozzi and especially S. Saint-Touch for perfect performance of quadratic RATs. The results of this work were reported by Z.B. at the Workshop INT-17-69W "Neutron Oscillations: Appearance, Disappearance, and Baryogenesis", Institute of Nuclear Theory, Seattle, USA, October 23-27, 2017, and at the seminar at Oak Ridge National Laboratory, Knoxville, USA, 31 Oct. 2017. We thank Klaus Kirch and Prajwal Mohan Murthy for informing us that a new experiment on search of $n-n^{\prime}$ oscillation was performed at the Paul-Scherrer Institute (PSI), Villingen, which results are currently under analysis.

Open Access This article is distributed under the terms of the Creative Commons Attribution 4.0 International License (http://creativecomm ons.org/licenses/by/4.0/), which permits unrestricted use, distribution, and reproduction in any medium, provided you give appropriate credit to the original author(s) and the source, provide a link to the Creative Commons license, and indicate if changes were made. Funded by SCOAP ${ }^{3}$.

\section{References}

1. T.D. Lee, C.N. Yang, Phys. Rev. 104, 254 (1956)

2. I.Y. Kobzarev, L.B. Okun and I.Y. Pomeranchuk, Sov. J. Nucl. Phys. 3(6), 837 (1966) [Yad. Fiz. 3, 1154 (1966)]

3. S.I. Blinnikov and M.Y. Khlopov, Sov. J. Nucl. Phys. 36, 472 (1982) [Yad. Fiz. 36, 809 (1982)]

4. E.W. Kolb, D. Seckel, M.S. Turner, Nature 314, 415 (1985)

5. M.Y. Khlopov et al., Sov. Astron. 35, 21 (1991) [Astron. Zh. 68, 42 (1991)]

6. R. Foot, H. Lew, R.R. Volkas, Phys. Lett. B 272, 67 (1991)

7. H.M. Hodges, Phys. Rev. D 47, 456 (1993)

8. Z. Berezhiani, A.D. Dolgov, R.N. Mohapatra, Phys. Lett. B 375, 26 (1996)

9. B. Holdom, Phys. Lett. B 166, 196 (1986)

10. S.L. Glashow, Phys. Lett. B 167, 35 (1986)

11. E.D. Carlson, S.L. Glashow, Phys. Lett. B 193, 168 (1987)

12. R. Foot, A.Y. Ignatiev, R.R. Volkas, Phys. Lett. B 503, 355 (2001)

13. Z. Berezhiani, A. Lepidi, Phys. Lett. B 681, 276 (2009)

14. R. Foot, H. Lew, R.R. Volkas, Mod. Phys. Lett. A 7, 2567 (1992)

15. E.K. Akhmedov, Z.G. Berezhiani, G. Senjanovic, Phys. Rev. Lett. 69, 3013 (1992)

16. R. Foot, R.R. Volkas, Phys. Rev. D 52, 6595 (1995)

17. Z. Berezhiani, R.N. Mohapatra, Phys. Rev. D 52, 6607 (1995)

18. Z.K. Silagadze, Phys. Atom. Nucl. 60, 272 (1997) [Yad. Fiz. 60N2, 336 (1997)] 
19. Z. Berezhiani, D. Comelli, F.L. Villante, Phys. Lett. B 503, 362 (2001). arXiv:hep-ph/0008105

20. A.Y. Ignatiev, R.R. Volkas, Phys. Rev. D 68, 023518 (2003). arXiv:hep-ph/0304260

21. Z. Berezhiani, P. Ciarcelluti, D. Comelli, F.L. Villante, Int. J. Mod. Phys. D 14, 107 (2005). arXiv:astro-ph/0312605

22. Z. Berezhiani, S. Cassisi, P. Ciarcelluti, A. Pietrinferni, Astropart. Phys. 24, 495 (2006). arXiv:astro-ph/0507153

23. L. Bento, Z. Berezhiani, Phys. Rev. Lett. 87, 231304 (2001). arXiv:hep-ph/0107281

24. L. Bento, Z. Berezhiani, Fortsch. Phys. 50, 489 (2002). arXiv:hep-ph/0111116

25. Z. Berezhiani, arXiv:1602.08599 [astro-ph.CO]

26. Z. Berezhiani, Int. J. Mod. Phys. A 19, 3775 (2004). arXiv:hep-ph/0312335

27. Z. Berezhiani, Eur. Phys. J. ST 163, 271 (2008)

28. Z. Berezhiani, in From Fields to Strings: Circumnavigating Theoretical Physics, eds. by Shifman, M. et al., 2005 vol. 3, (World Scientific), pp. 2147-2195. https://doi.org/10.1142/ 9789812775344-0055 arXiv:hep-ph/0508233

29. L.B. Okun, Phys. Usp. 50, 380 (2007). arXiv:hep-ph/0606202

30. Z. Berezhiani, Phys. Lett. B 417, 287 (1998)

31. A. Addazi, Z. Berezhiani and Y. Kamyshkov, Eur. Phys. J. C 77(5), 301 (2017). arXiv:1607.00348 [hep-ph]

32. Z. Berezhiani, Eur. Phys. J. C 76(12), 705 (2016). arXiv:1507.05478 [hep-ph]

33. S.N. Gninenko, Phys. Lett. B 326, 317 (1994)

34. S.N. Gninenko, N.V. Krasnikov, V.A. Matveev, A. Rubbia, Phys. Part. Nucl. 37, 321 (2006)

35. A. Badertscher et al., Phys. Rev. D 75, 032004 (2007). arXiv:hep-ex/0609059

36. P. Crivelli et al., JINST 5, P08001 (2010). arXiv:1005.4802 [hepex]

37. C. Vigo et al., Phys. Rev. D 97(9), 092008 (2018). arXiv:1803.05744 [hep-ex]

38. R. Foot, Phys. Rev. D 86, 023524 (2012). arXiv:1203.2387 [hep$\mathrm{ph}]$

39. R. Cerulli, et al., Eur. Phys. J. C 77(2), 83 (2017). arXiv:1701.08590 [hep-ex]

40. A. Addazi, et al., Eur. Phys. J. C 75(8), 400 (2015). arXiv:1507.04317 [hep-ex]

41. Z. Berezhiani, L. Kaufmann, P. Panci, N. Rossi, A. Rubbia and A.S. Sakharov, CERN-PH-TH-2008-108

42. Z. Berezhiani, L. Bento, Phys. Rev. Lett. 96, 081801 (2006). arXiv:hep-ph/0507031

43. Z. Berezhiani, Eur. Phys. J. C 64, 421 (2009). arXiv:0804.2088 [hep-ph]
44. M. Baldo-Ceolin et al., Z. Phys. C 63, 409 (1994)

45. D.G. Phillips et al., Phys. Rep. 612, 1 (2016). arXiv:1410.1100 [hep-ex]

46. K.S. Babu et al., "Neutron-Antineutron Oscillations: A Snowmass 2013 White Paper," arXiv:1310.8593 [hep-ex]

47. Z. Berezhiani, L. Bento, Phys. Lett. B 635, 253 (2006). arXiv:hep-ph/0602227

48. Z. Berezhiani, A. Gazizov, Eur. Phys. J. C 72, 2111 (2012). arXiv: 1109.3725 [astro-ph.HE]

49. Z. Berezhiani, R. Biondi and A. Gazizov, in preparation

50. R.N. Mohapatra, S. Nasri, S. Nussinov, Phys. Lett. B 627, 124 (2005). arXiv:hep-ph/0508109

51. A. Coc, J.P. Uzan and E. Vangioni, Phys. Rev. D 8712, 123530 (2013). arXiv:1303.1935 [astro-ph.CO]

52. A. Coc, M. Pospelov, J.P. Uzan and E. Vangioni, Phys. Rev. D 90(8), 085018 (2014). arXiv:1405.1718 [hep-ph]

53. Z. Berezhiani and A. Vainshtein, arXiv:1506.05096 [hep-ph]

54. Z. Berezhiani, R. Biondi, Y. Kamyshkov and L. Varriano, in preparation

55. Y.N. Pokotilovski, Phys. Lett. B 639, 214 (2006). arXiv:nucl-ex/0601017

56. G. Bison et al., Phys. Rev. C 95(4), 045503 (2017). arXiv:1610.08399 [physics.ins-det]

57. G. Ban et al., Phys. Rev. Lett. 99, 161603 (2007). arXiv:0705.2336 [nucl-ex]

58. A.P. Serebrov et al., Phys. Lett. B 663, 181 (2008). arXiv:0706.3600 [nucl-ex]

59. K. Bodek et al., Nucl. Instrum. Methods A 611, 141 (2009)

60. A.P. Serebrov et al., Nucl. Instrum. Methods A 611, 137 (2009). arXiv:0809.4902 [nucl-ex]

61. I. Altarev et al., Phys. Rev. D 80, 032003 (2009). arXiv:0905.4208 [nucl-ex]

62. C. Patrignani et al., [Particle Data Group], Chin. Phys. C 40(10), 100001 (2016)

63. Z. Berezhiani, F. Nesti, Eur. Phys. J. C 72, 1974 (2012). arXiv: 1203.1035 [hep-ph]

64. A.Y. Ignatiev, R.R. Volkas, Phys. Rev. D 62, 023508 (2000). arXiv:hep-ph/0005125

65. Z. Berezhiani, A.D. Dolgov, I.I. Tkachev, Eur. Phys. J. C 73, 2620 (2013). arXiv:1307.6953 [astro-ph.CO]

66. R. Biondi, arXiv:1803.03522 [physics.comp-ph]

67. A. Steyerl et al., Phys. Lett. A 116, 347 (1986)

68. R. Golub, J.M. Pendlebury, Rep. Prog. Phys. 42, 439 (1979)

69. Z. Berezhiani et al., Phys. Rev. D 96(3), 035039 (2017). arXiv:1703.06735 [hep-ex]

70. L.J. Broussard et al., arXiv:1710.00767 [hep-ex] 